\title{
Are there any factors influencing the course of multistage treatment in Hirschsprung's disease?
}

\author{
Patrycja Sosnowska, Michał Błaszczyński, Sebastian Moryciński, Witold Porzucek, Przemysław Mańkowski \\ Department of Paediatric Surgery, Traumatology, and Urology, Poznan University of Medical Sciences, \\ Karol Jonscher University Hospital, Poznan, Poland
}

Gastroenterology Rev 2016; 11 (2): 131-135 DOI: $10.5114 / p g .2016 .57772$

Key words: child, intestinal obstruction, surgical stomas.

Address for correspondence: Patrycja Sosnowska MD, Department of Paediatric Surgery, Traumatology, and Urology, Karol Jonscher University Hospital, 27/33 Szpitalna St, 60-572 Poznan, Poland, phone: +48 61849 15 78, +48 510094 296, fax: +48 6184752 28, e-mail: patrycja.sosnowska@outlook.com

\begin{abstract}
Introduction: Surgical treatment of Hirschsprung's disease may be performed in a single step, or in stages with a temporary stoma. The therapy depends on the clinical condition of the patient and the severity of symptoms. Planned multistage treatment is carried out in two or three steps.

Aim: To analyse our 15 years of experience with multistage surgery for the treatment of Hirschsprung's disease, to identify multistage-related factors influencing the course of surgery and hospitalisation, and to evaluate the probability of complications during multistage treatment.

Material and methods: The study material was collected on the basis of documentation of patients treated during the years 2000 to 2014. The parameters concerning surgery and hospitalisation were statistically analysed.

Results: Twenty nine patients were treated with multistage surgery using the following methods: Duhamel-Martin and Transanal Endorectal Pull-Through (TEPT). There were significant correlations $(p<0.05)$ between length of resected intestine and operative time. Classification and Regression Tree (CRT) was used to classify the operated children depending on the presentence of complications after surgery.

Conclusions: The incidence of complications during multistage treatment for both methods was comparable. It is difficult to objectively compare the Duhamel-Martin and TEPT techniques because of the different indicators for their use in multistage surgery. Intestinal adhesions were the most common complication after definitive surgery. Younger age of the operated patients was associated with greater risk of adhesion formation.
\end{abstract}

\section{Introduction}

There are two methods of treatment for patients with Hirschsprung's disease. The surgery can be performed in a single step, or in stages using temporary intestinal stoma. The strategy depends on the clinical condition of the patient and the severity of symptoms. Patients with emergent complications of disease have been treated with exteriorisation of the intestine as a stoma. After stabilisation of the patient's condition, control of enterocolitis, and at least partial normalisation of megacolon, the aganglionic segment is removed and the stoma is closed, either at the time or later.

Planned staged treatment is carried out in two or three steps. If surgery treatment is performed in two stages the first step is exteriorising the intestine as a stoma, the second stage is definitive surgery (removal of the aganglionic intestine) and closing the stoma. The continuity of the digestive system is restored. When the role of the stoma is protection after radical surgery and distal anastomosis is done, the stoma closure is performed about 6 months later during the third stage [1].

Various surgical methods were described as the ways to reconstruct the anorectal area in Hirschsprung's disease treatment. There are included in classical surgeries: Duhamel's, Swenson's, Soave's, Rehbein's and the more recently introduced and currently widely performed transanal endorectal pull-through (TEPT) technique $[2,3]$.

Patients operated due to Hirschsprung's disease have different outcomes after radical surgery. Some children experience complications in the postoperative 
period and sometimes surgical intervention has to be taken into consideration [4].

\section{Aim}

The purpose of the study was to analyse our 15 years of experience in multistage surgery for the treatment of Hirschsprung's disease, to identify multistage-related factors influencing the course of surgery and hospitalisation, and to evaluate the probability of complications in multistage treatment.

\section{Material and methods}

During 15 years of the study, from 2000 to 2014, 29 patients were treated using multistage surgery methods, in the Department of Paediatric Surgery, Traumatology, and Urology in Poznan. Five girls and 24 boys were operated on. The Duhamel-Martin and TEPT techniques were used. Final diagnosis of Hirschsprung's disease was confirmed by biopsy and histopathology in all patients. None of the patients was omitted from the study. Patients referred from other centres by first diagnosis and colostomy were not included in the study. The choice of 2 or 3 stages was made by the surgeon and it was connected with the surgical condition of the patient. During radical surgery the level of intestinal resection was determined basing on intraoperative histopathology examination.

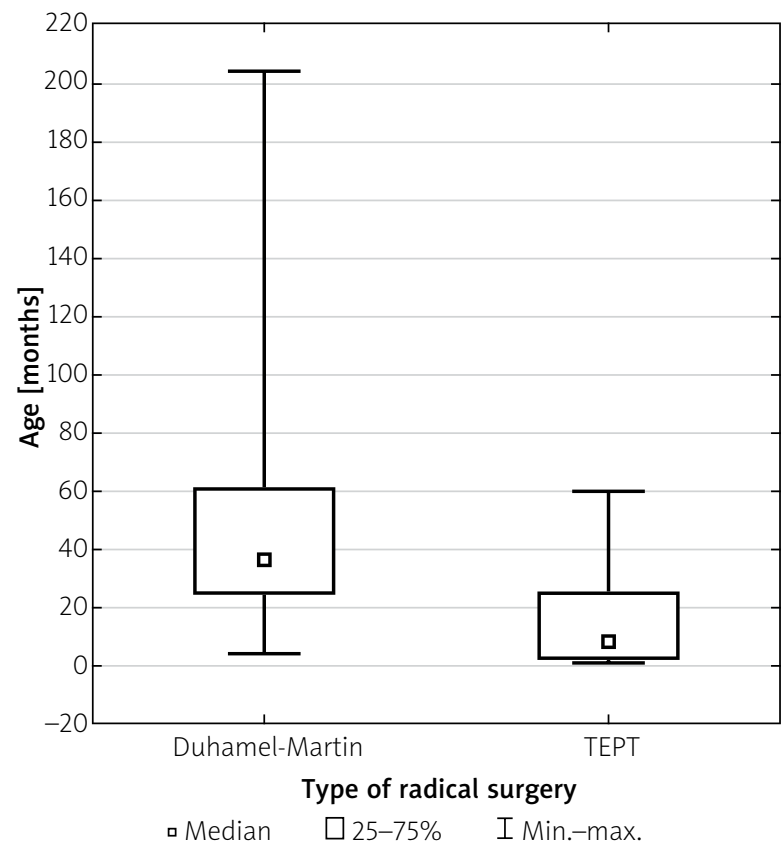

Figure 1. Boxplot presenting the median age of patients radically operated on using Duhamel-Martin and TEPT techniques
Prophylactic antibiotic therapy was initiated on the day of the surgery and continued during hospitalisation. It was chosen conforming to current internal guidelines issued by the hospital and was applied during each surgery.

The analysis included the following parameters: technique of radical surgery, age of patient (at the time of definitive surgery), length of resected intestine, operative time of radical surgery, length of hospitalisation after radical surgery, number of planned stages of surgical treatment, number and type of stomas, number of all performed surgeries, number and cause of complications during staged treatment.

The study was conducted in accordance with a protocol presented to the Bioethical Commission of Poznan University of Medical Sciences (Decision No. 58/12).

\section{Statistical analysis}

The results were collected to analyse the parameters and to classify patients according to the analysed characteristics. Calculations were performed using Statistica 10 (StatSoft Inc., Tulsa, USA). The statistical significance was set at $p<0.05$. To examine the relationship between the described variables the Mann-Whitney's test and the Spearman's rank correlation coefficient were used.

In order to classify patients to the appropriate group, due to the presence of complications after radical surgery of Hirschsprung's disease, the data mining method of Classification and Regression Tree (CRT) was used. Its construction included the age of the patient and the number of stomas. The CRT exhaustive search for univariate splits method was used to find divisions of the predictor variable. The Gini measure was chosen as the measure of goodness of fit. The FACT (Fast Algorithm for Classification Trees)-style direct stopping was used as the stopping rule.

\section{Results}

Duhamel-Martin surgery was used in 19 children and the TEPT technique in 10 patients. The average period from exteriorising stoma to radical surgery was 5 months. The shortest and the longest times between these surgeries were 3 and 8 months, respectively. Analysis of the results showed that the average age of patients operated radically during multistage treatment was 38 months: 49 months for Duhamel-Martin surgery and 16 months for TEPT technique. Children were operated in the age range 1 month to 17 years of age. Analysis of the results found a correlation between the age of the patient and the type of radical surgery (Figure 1).

The average length of resected intestine was $37 \mathrm{~cm}$ : $46 \mathrm{~cm}$ for the Duhamel-Martin technique and $18 \mathrm{~cm}$ for TEPT surgery. The next correlation found was the length 


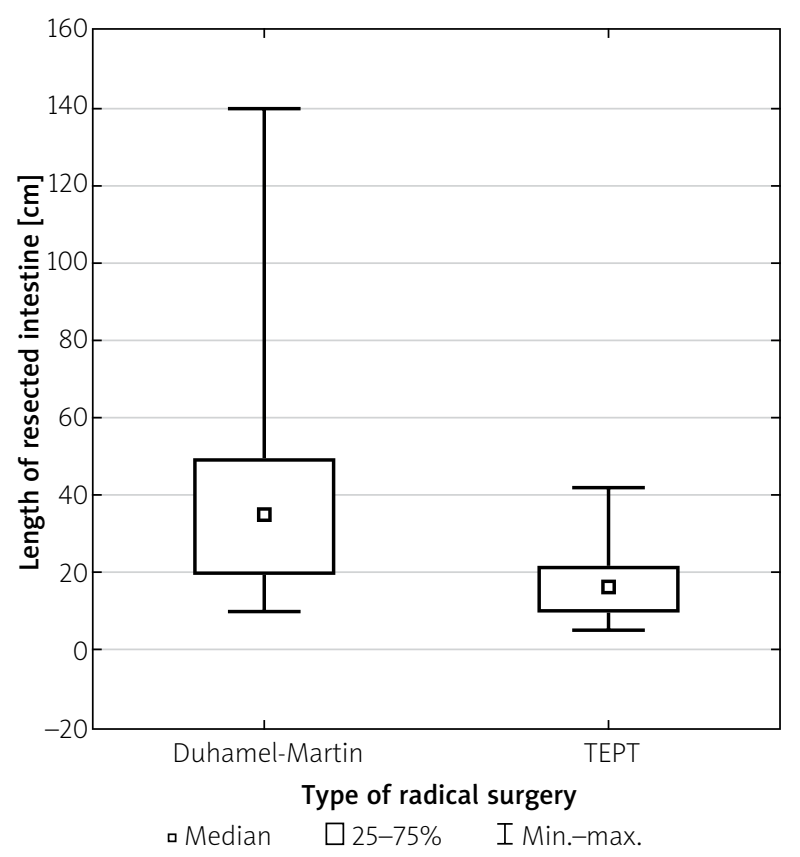

Figure 2. Boxplot presenting the median length of resected intestine of patients radically operated on using Duhamel-Martin and TEPT techniques

of the resected intestine and the type of radical surgery (Figure 2).

The mean time of radical surgery was four hours for the Duhamel-Martin method. The shortest and the longest times of this operation were 2 and $6 \mathrm{~h}$, respectively. Patients treated TEPT surgery were operated on average for $3 \mathrm{~h} 50 \mathrm{~min}$. The shortest and the longest times in this technique were $2 \mathrm{~h}$ and $4 \mathrm{~h} 30 \mathrm{~min}$, respectively. The analysis of the results also showed the correlation between length of resected intestine and operative time of radical surgery (Figure 3 ).

The average time from radical surgery to discharge the child from the hospital was 17 days for both methods. The shortest and the longest times of hospitalisation were 9 and 60 days, respectively, for Duhamel-Martin technique and 7 and 40 days, respectively, for TEPT surgery.

Analysing the whole therapeutic process, $13 \mathrm{pa}$ tients had 2 surgeries, 10 children had 3 surgeries, 5 children had 4 surgeries, and 1 patient had 5 surgeries, during multistage treatment.

Two-stage surgery was used in 14 patients operated with Duhamel-Martin technique and in 6 patients treated with TEPT surgery. Three-stage surgery was performed in 5 children operated using Duhamel-Martin technique and in 4 patients treated TEPT surgery. Seventeen patients had 1 colostomy, 9 had 2 colosto-

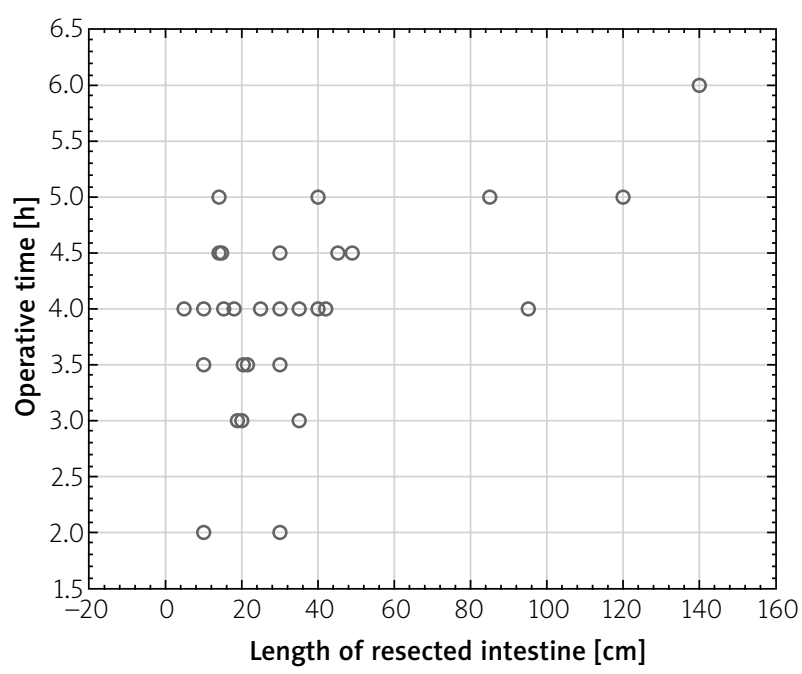

Figure 3. Scatterplot illustrating the correlation between the length of resected intestine and the length of operative time in patients operated on using Duhamel-Martin and TEPT techniques

mies, and 3 patients had 1 ileostomy, during multistage treatment. Our study showed that the number of stomas and number of surgeries did not have an influence on the course of radical surgery or on the incidence of complications.

Two patients developed occlusive symptoms after radical surgery, but conservative treatment was sufficient. The remaining 8 patients with obstruction needed laparotomy. Three of these children had 2 laparotomies, and 1 patient had 3 laparotomies. Adhesiotomy was performed in every case. Enterocolitis was diagnosed in 2 patients. Broad-spectrum antibiotic therapy was used in one child. For this patient the symptoms have subsided. The second child needed additional laparotomy with bowel diversion. Prolapse of stoma was noted in four cases. One child had to be operated on for this reason. During the study no side effects like itches or wound infection were seen in the group. Stool incontinence was noted in 1 patient, which was cured after surgery by conservative medical treatment (Table I).

Table I. Postoperative complications during staged treatment

\begin{tabular}{lc} 
Complications & Number of patients (\%) \\
\hline Obstruction & $10(34)$ \\
\hline Stoma prolapse & $4(14)$ \\
\hline Enterocolitis & $2(6)$ \\
\hline Stool incontinence & $1(3)$
\end{tabular}




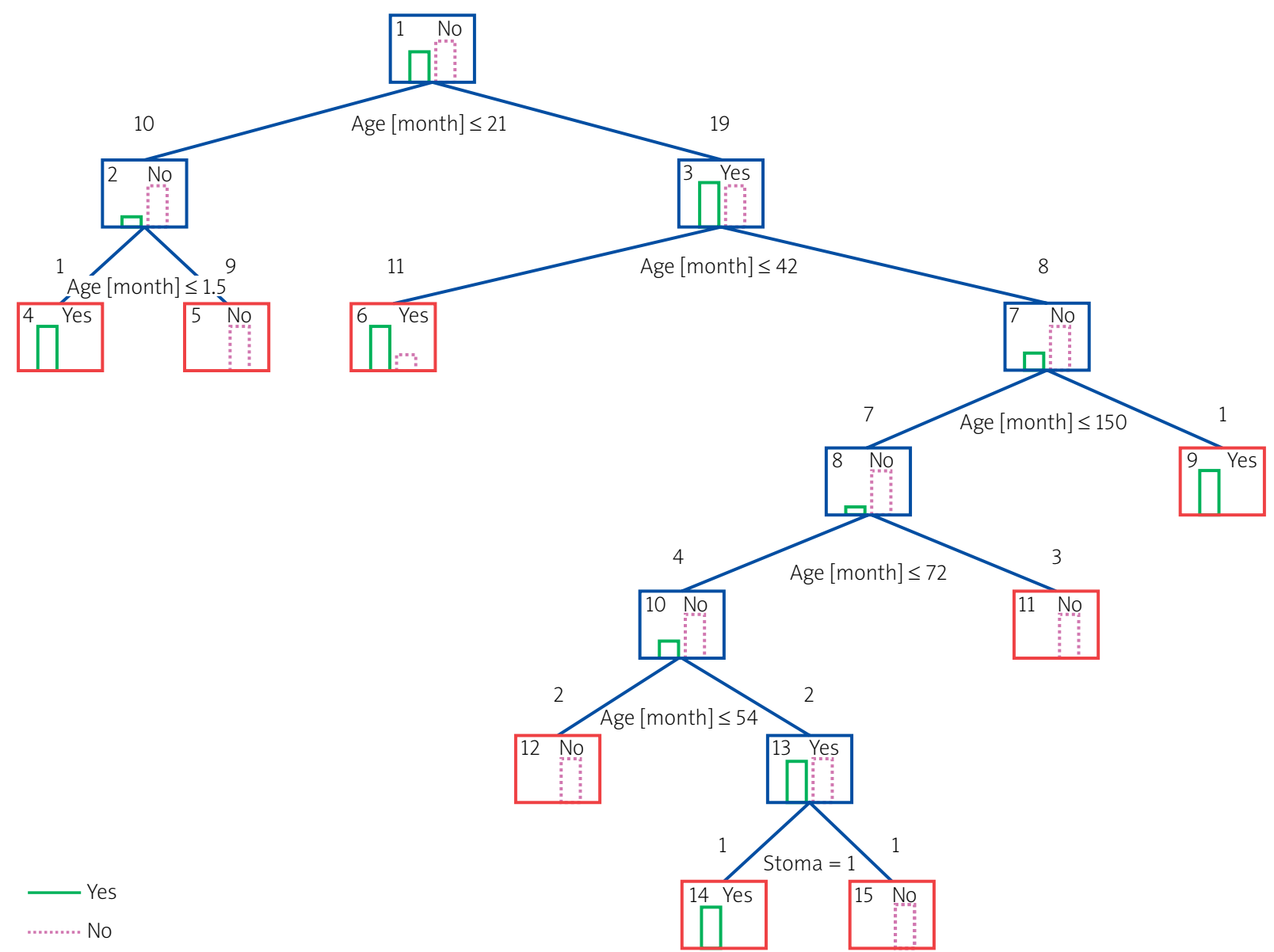

Figure 4. CRT showing the classification of children due to the presence of complications after surgical treatment of Hirschsprung's disease

The analysis of the results additionally found the correlation between the probability of complications and the age of radical surgery (Figure 4).

The CRT analysis shows that most of complications occurred in children between 21 and 42 months of age.

\section{Discussion}

Hirschsprung's disease is one of the commonly analysed diseases among researchers in paediatric surgery. This condition was first described by Harald Hirschsprung as a congenital megacolon in 1888. Since then various methods for diagnosis and treatment of Hirschsprung's disease have been introduced [5].

Colostomy was a standard part of the treatment of this disease in the past. This was aimed at control of enterocolitis, at least partial normalisation of megacolon, decompression of the gastrointestinal tract, and protection of the performed anastomosis [3]. Colostomy decreases the risk of anastomotic leak and pelvic abscess, and also allows for smooth healing. All these arguments mean that multistage treatment still has its followers.
First of all, multistage treatment is the method of choice when primary definitive surgery is impossible because of the clinical condition of the patient.

This study shows correlations between parameters describing the surgical therapeutic process and presents classification of patients to appropriate groups, analysing the incidence of complications, taking into account the age at the time of radical surgery and the number of ostomies.

Analysing the Duhamel-Martin and TEPT techniques, the average age of radical surgery was higher for children operated with Duhamel-Martin technique; it was 49 months (4 years) vs. 16 months for TEPT (Figure 1). The use of Duhamel-Martin surgery in small children is limited by the size of disposable linear and circular staplers. The best option would be use of a laparoscopic stapler, but this was not the practice in our centre [6].

The average length of resected intestine was more than two times longer for Duhamel-Martin technique compared to TEPT (Figure 2). This situation is not directly related to the technique of surgery and resulted 
from the length of aganglionic segment necessary for resection. If the length of resected intestine was longer, it was associated with a longer time of surgery. It is associated with more difficult and longer dissections (Figure 3).

In our centre the planned multistage treatment was performed in 2 or 3 stages. The incidence of early and late complications during two- or three-stage treatment was the same. A comparable frequency of postoperative complications (additionally comparing one-stage surgery) is also described by Holschneider [7].

In our study adhesive intestinal obstruction was the most common complication after radical surgery and occurred in 10 (34\%) children. Eight children did not respond to conservative treatment and underwent surgical treatment. The incidence of postoperative bowel obstruction can be decreased using laparoscopically assisted procedures, which is recommended by its proponents [7].

The second most common complication was prolapse of stoma, which occurred in 4 patients. The incidence of stomal complications referred in literature by other authors is the same for patients with Hirschsprung's disease and for other diseases [7, 8]. Enterocolitis and stool incontinence were described sporadically, and occurred, respectively, in $6 \%$ and $3 \%$ of children. The incidence of enterocolitis after radical surgery of Hirschsprung's disease ranges from $6 \%$ to $42 \%$ and varies widely in the literature depending on the study $[7,9,10]$.

Faecal soiling has a negative influence on the quality of life in children with Hirschsprung's disease. This complication occurred in 1 patient in our series and lasted for 3 years after surgery performed at the age of 3 years. Rescorla et al. noted that between 10 and 15 years of age the incidence of incontinence declined, and in patients older than 15 years they did not observe incontinence [11].

The aim of CRT was to classify patients into two groups, due to the presence of complications after radical surgery. Most of the complications occurred between 21 and 42 months of age (Figure 4). These patients accounted for $37 \%$ of all children. The most common causes of complications were intestinal adhesions, as described above. Such classification confirms the fact that young age at the time of surgery may be related to a higher risk of intestinal adhesions [12].

\section{Conclusions}

Summing up, the condition of the patient, age at the time of surgery, and length of resected intestine have an influence on the course of multistage treatment of Hirschsprung's disease. Younger age of operated pa- tients was always associated with greater risk of formation of intestinal adhesions. It was the most common complication after radical surgery. The most commonly used technique in multistage surgery in the treatment of Hirschsprung's disease was the Duhamel-Martin procedure. It is difficult to objectively compare the Duhamel-Martin and TEPT techniques because of different indicators for their use in multistage surgery. The incidence of complications during multistage treatment for both methods was comparable.

\section{Conflict of interest}

The authors declare no conflict of interest.

\section{References}

1. Ghoroubi J, Imanzadeh F, Sadeghian N, et al. Comparison between one and multiple stages surgery in the treatment of Hirschsprung's' disease. Ann Pediatr Surg 2009; 5: 172-6.

2. Romero P, Kroiss M, Chmelnik M, et al. Outcome of transanal endorectal vs. transabdominal pull through in patients with Hirschsprung's disease. Lagenbecks Arch Surg 2011; 396: 1027-33.

3. Stringer M, Oldham K, Mouriquand P. Pediatric surgery and urology: long-term outcomes. Cambridge University Press 2006; 385-6.

4. Parithan P, Chiengkriwate P, Chowchuvech V, et al. Bowel preparation for pull-through operation in Hirschsprung's disease. Songkla Med J 2007; 25: 401-6.

5. Zain A, Fadhil S. Hirschsprung's disease: a comparison of SWenson's and Soave's pull-through methods. Iraqi J Med Sci 2012; 10: 69-74.

6. Sosnowska P, Blaszczyński M. A 15-year experience with the one-stage surgery for treatment of Hirschsprung's disease in newborns, infants, and young children. Indian J Surg 2015; 77 (Suppl 3): 1109-14.

7. Holschneider A. Hirschsprung's disease and allied disorders. Springer, Berlin 2006.

8. Nour S, Beck J, Stringer M. Colostomy complications in infants and children. Ann R Coll Surg Engl 1996; 78: 526-30.

9. Surana R, Quinn F, Puri P. Evaluation of risk factors in the development of enterocolitis complicating Hirschsprung's disease. Pediatr Surg Int 1994; 9: 234-6.

10. Hackam D, Filler R, Pearl R. Enterocolitis after the surgical treatment of Hirschsprung's disease: risk factors and financial impact. J Pediatr Surg 1998; 33: 830-3.

11. Rescorla F, Morrison A, Engles D, et al. Hirschsprung's disease: evaluation of mortality and long-term function in 260 cases. Arch Surg 1992; 127: 934-41.

12. Piaseczna-Piotrowska A, Jóźwiak A. Niedrożność zrostowa jelit po operacjach brzusznych u dzieci - analiza 94 przypadków. Med Wieku Rozwoj 2011; 15: 91-5.

Received: 8.01.2015

Accepted: 5.05.2015 